\title{
Incorporating Live Action into the CALL Lab
}

\section{Elizabeth Hanson-Smith}

Computer-assisted technology, even in its infancy as a medium, has proven to enhance language learning, sometimes in unexpected ways (see the early RAND study, Glennan \& Melmed 1996). Even the most traditionally designed "drill-and-grill" exercises can

- Allow for differing learning styles, including those of special needs students;

- Offer voices different from the teacher's, an especially important advantage in a non-native speaker environment;

- Use multiple input channels to stimulate better recognition, recall, and affective receptiveness;

- Open a window on cultural practices in typical settings and activities;

- Empower students to take control of their own learning processes, and hence take more personal responsibility for learning;

- Provide far more individuated practice than can a harried classroom teacher with 30 or more students.

However, as pointed out by Roche (1999) many language learning CDs either are dominated by an audio-lingual or grammar-translation mentality, perhaps formed by the assumptions and prejudices of programmers, or are confined by the limitations of the computer medium itself. Internet-based language learning Websites suffer from similar failings. Computers are basically dumb machines and can do only what the programmers have asked them to do, and often programmers are not linguistically trained language teaching specialists. Even more telling, perhaps, is that computers are not yet capable of handling natural language in all its variety and richness, nor may they ever be. Given these strictures, what are some of the ways to best use technology in language teaching and learning?

Live Action English Interactive (and its sister product, Live Action Spanish Interactive) is one of the few language learning CDs to be designed and programmed solely by practicing teachers-with a combined classroom experience of over 100 years in the field of language education. It is also one of the few language products to be informed by a well recognized approach to language teaching, Total 
Physical Response (see Asher 1996), which was referred to by Krashen and Terrell as an example of the so-called Natural Approach to second language acquisition (see Krashen 1982, and Krashen \& Terrell 1983).

In the Total Physical Response (TPR) approach, students are asked to engage in physical activities, responding to commands before they are expected to speak. The teacher may begin by demonstrating actions while repeating the appropriate short imperatives in a sequence: Turn on the water. Pick up the soap, Wash your hands, etc. (Romijn and Seely 2002, p.1). Students then perform the actions, while listening to the commands before attempting to speak themselves. The idea is to build listening comprehension of sentences related meaningfully to physical actions, people, and objects in context; the kinesthetic aspect is not only motivating, but enhances the memorability of the meanings and may match closely the preferred learning styles of many students, particularly younger learners. However, adults invariably enjoy the activity as much as children do, and benefit from the prolonged "silent period," as proponents of comprehension-based learning recommend (see for example, Krashen 1982). The pre-production silence allows students to hear language input repeatedly and learn to understand meanings while acting them out. Contextualization in familiar actions and appropriate sequences aids comprehension and the formation of meaningful associations, while the silent period contributes to better pronunciation and grammar when learners begin to speak.

Students at first hear only the imperative verb forms (which in many languages are the "root" of the other forms); eventually they are allowed to use the commands with others: Jorge, open the can, or Chen, take off the lid. One of the difficulties of language learning is receiving enough input enough times to grasp meanings while acquiring grammatical structures-all without becoming bored (often the problem with aural-oral repetition). Physical action, the acting out or miming of meaningful activities, naturally involves the brain in creating dense associations and improves memory, concentration, and motivation. (See Asher 1983, for research studies of the effects of TPR and comprehension-based language acquisition.)

Eventually students build increasingly complex sentence structures, leading to narrative structures with the conjugated verb forms that require the various pronouns and tenses: Yesterday, I opened a can and poured the soup into a pot. Students meanwhile are acquiring a fairly large vocabulary of common objects and actions in familiar everyday situations, tense sequences, and (especially difficult in English) various two-part verbs and prepositional locutions. (For more on the theory of TPR, see Romijn \& Seely 1998, and Ray \& Seely 1998.) The use of sentences, rather than the memorization of 
individual vocabulary items, not only gives learners a large mental database of words and expressions, but also entails a great deal of repetition, which aids the formation of what some professionals would call automaticity. TPR has spread across the globe and is now used in language classes from Europe to Asia. (See Yeh 2004, for videos of live classroom sessions with her teachers-in-training in Taiwan; and González \& Mühren 2004, for a recent video- and Websupported conference presentation on TPR in Spain and The Netherlands.)

It may seem somewhat paradoxical to attempt to transform TPR, which is kinesthetically-based, into a computer program that students will use while sitting at a workstation; however, Live Action English Interactive (LAEI) and Live Action Spanish Interactive (LASI) are among the few beginner-intermediate level language CDs designed to be used in close coordination with TPR classroom activities. Although they can be used for self-study, the CDs are intended neither as a replacement for the classroom teacher nor as a stand-in for the whole curriculum, but rather as a supplement for additional practice in a blended (face-to-face and electronic) environment. Nine of the 12 units on the CDs are taken directly from scripts in the texts, Live Action English (Romijn \& Seeley 2002) and ¿Viva la Acción! Live Action Spanish (Seely and Romijn, 2001), while three additional units were created for their particular appropriateness for the computer medium (Sending a Postcard, Planting a Seed, and Going Fishing). Since the LASI CD is based on the LAEI program, with certain adjustments to provide authenticity for the Spanishlearning audience, references in most of the remainder of this article will be to LAEI, which targets adult learners of English as a second or foreign language. I will speak of some of the decisions made about LASI in a separate section below. This paper will discuss the process of creating the $\mathrm{CD}$ and describe in some detail the resultant product, including both its significant features and the compromises that had to be made in adapting this approach to teaching and learning into the electronic medium. It is hoped that this information will help teachers planning similar projects or evaluating language-learning software for their own language programs. 
The Process
LAEI began when Robert Wachman, a community college teacher of English as a Second Language (ESL) and former Peace Corps volunteer who had used TPR over the years, experimented with HyperCard authoring software to transform scripts from Live Action English (LAE) into short presentations with line drawings from Action English Pictures (based on LAE, Takahashi and FraumanPrickel 1999). Joined in his experiments by Elizabeth HansonSmith, a CALL consultant, ESL teacher trainer, and software designer (Oxford Picture Dictionary Interactive), Wachman contacted the authors of LAE, Contee Seely and Elizabeth Romijn, and proposed a $C D$ version. An experienced teacher and CALL program author, Larry Statan, was quickly persuaded to join the group. Statan, designer of such programs as All Clear! and Making Connections, suggested a more sophisticated authoring program, Macromedia's Director (2004), be used because of its professional look and feel, its cross-platform capability (both Apple and PCs), and its several built-in features that were useful for language learning (these will be mentioned in the Product section below). Statan quickly brought the simple exercises to life with a combination of video, morphed images, and animated photographs. He also designed ingenious practice activities that allow the student to control objects and video on the computer screen, a form of virtual physical response, which will be described further below.

Some of the most important design decisions had to be made very early in the process. In turn, many of these decisions had to be based on assumptions about the students who would use the program:

-What age are the target students?

- How much computer expertise would they need to use the program?

- Would they have access to a lab or use the $C D$ at home?

- Would they have access to headphones and microphones? Other decisions had to be based on the limitations of the computer medium:

- How much content could be included in the program?

- How many CDs would be required? Should we do one or two?

- How could the content of the TPR books best be translated into multimedia? Should we use drawings? Photos? Video? Speech recognition?

- Should our English learners be instructed in computer and program use? 
And finally, decisions about the content itself had to be made before authoring could begin:

-Was this to be a tutorial intended to replace the teacher?

- Was it additional practice? Or some combination of these two?

- What kinds of reward system and feedback would be given for correct or incorrect responses?

- Should there be a testing component?

- How would teachers know what effort the students had put into using the $C D$ ? That is, would there be a scoring system?

Several factors were of importance during the design process: although all five of us taught at a wide variety of levels, all had used TPR in one form or another and agreed fully on its value as an approach to teaching. Another major advantage was the inclusion on the team of people with experience in programming, computer instructional design, and $C D$ production who were also classroom teachers. We had a compatible understanding of how and why to use computer technologies in language learning, and had no illusions about the computer ever replacing the teacher. We believed, however, that the electronic medium provided the perfect place to give students extensive practice, practice well beyond what a single teacher in a classroom of 15-30 students could ever do individually. We also had no need to consult with marketing experts, editorial staff, or advertising agencies: we would use the product ourselves and assume that other teachers would find it of equal value and interest. Presentations at professional conferences and word-of-mouth have been our chief means of promotion.

Since most of us were teaching adult learners in community colleges or adult education classes for immigrants, often held in community centers or high schools at night, and since TPR was highly appropriate to the beginning-intermediate learner, this became our target population. Given the usual financial circumstances of this population, and their lack of computer expertise, we felt that the technology should be as simple as possible to operate. Students would practice the basics of mousing and keyboarding and learn these as they used the program. We have since learned that special needs students also find the program appropriate and accessible. Since most of the locations where adult learners would use the software (e.g., libraries, community centers, and high school after-hours labs) had networked labs or several stand-alone workstations, using CALL in itself would not present a problem as long as the technology was simple and appropriate. And finally, while technology might provide 
some novelty, we knew the program had to be intrinsically motivating to the students so that they would keep using it repeatedly, and obtain from it enough practice to acquire language.

A potential problem we especially intended to avoid-one glaringly evident in some purportedly "adult ed" programs-is the juvenilization of the beginner-intermediate content and interface. Just because these are beginners in language does not mean our adult learners are child-like.

Once these basics were decided upon, the group met in person infrequently, but online through e-mail messaging quite often. We quickly agreed upon a prototype menu designed by Statan (see Figure 1), and he began the authoring process. As work progressed, we all conferred frequently on the various units. The ease of online file transfer and the discussion of content through e-mail made the process very time-efficient. It was also helpful to feel that as a group we not only thought along similar lines pedagogically, but also respected and liked each other as well. Some important elements to be learned from this process are

- Make sure the team is compatible on a personal and professional level

- Make sure all team members believe in and are committed to the pedagogical theory that underlies the product

- Make sure there is early agreement about the audience and goals of the project

- Don't let distance interfere with team communications-a world-wide team can be assembled electronically

- Keep in mind that design decisions have to made very early in the process-better to spend more time in planning beforehand than revising the project later. 


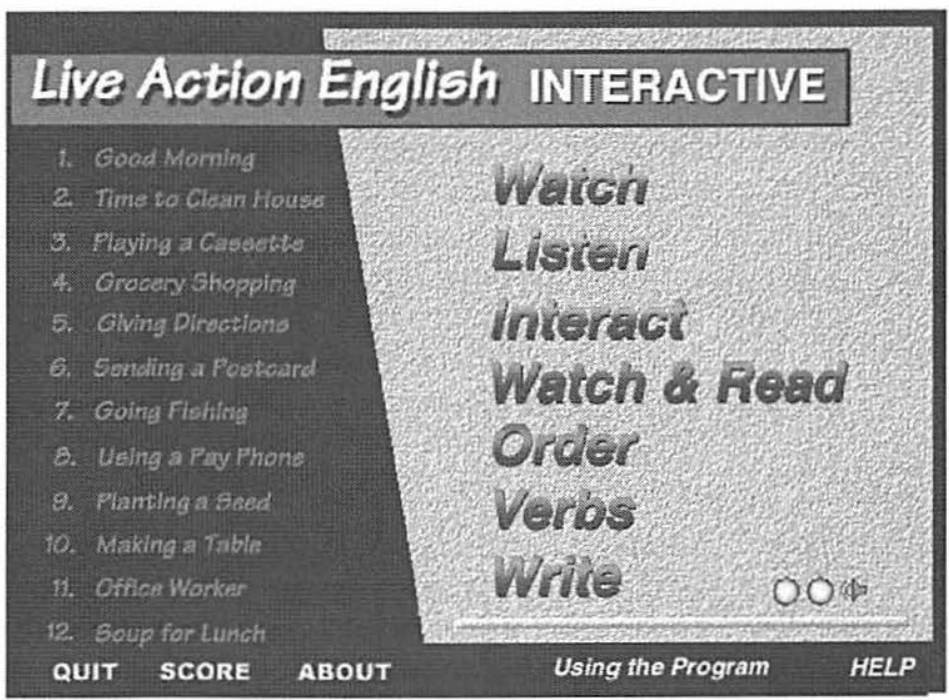

\section{The Product}

Figure 1. The Main Menu screen of Live Action English Interactive (Command Performance Language Institute 2000) offers access to all units with a minimum number of clicks. Most units are tied to similarly titled units in the Live Action English text (Romijn and Seely 2002).

One of our initial decisions concerned the type of media to use within the $\mathrm{CD}$. While our prototype had used animated black line drawings, crudely colorized, Statan suggested that video clips would offer far more realistic representations of contemporary life in the U.S. In the classroom, students had to imagine the contexts of the TPR scenarios, but with the CD, they could view a true-to-life scene. Because of space limitations on the $\mathrm{CD}$, Statan filmed some units totally or partially with a digital video camera, and shot others in still photos (with occasional morphing), but with movement implied through the sequencing of the images. Interestingly, students, when questioned after using a unit, are unaware of which technology has been employed, presumably because they are so focused on the language presentation and settings.

While it was tempting to use speech or voice recognition technology so that students could speak to the computer and possibly receive a simulated response, we perceived several problems: First, it was felt that hearing the native-speaking models on the $C D$ repeatedly would provide better input than having students listen to their own voices. This is also a tenet of the TPR approach: learners need input far more than output in the initial learning stages. Computer technology is also not at a point where it can easily elicit and correct pronunciation. The computer might give an approximate guess at what 
the student was saying or allow students to compare their speech to a model, but computers cannot yet provide satisfactory correction to natural utterances. Secondly, it is not yet easy to provide voice or speech recognition technology that would work with all the different types of possible input devices our students might use. Third, our intended students, adult learners, might not have the necessary equipment to use or an appropriate space to use it in. We could not expect adult learners to go out and invest in microphones or specific sound cards. Additionally, public access points, such as libraries, community centers, or Internet cafes, would not have microphones and would not encourage speaking out loud. Speech or voice recognition could be a problem in noisy school labs where students would have to compete to be heard.

Another design decision was to make the CD-ROM product as portable and accessible as possible. This meant a single, stand-alone $\mathrm{CD}$ would be produced. There is no installation or initialization process required (another advantage of excluding voice recognition); the student could run the program directly from the $C D$, which meant the product could be checked out from a library and taken home on loan, or copied directly to a hard drive on a single workstation, or run from a server in a networked lab. Naturally, we would have to depend on the honesty of the purchaser and the end user in respecting copyright. This decision eliminated the need for an elaborate password secured system that might create problems for student users, and for teachers who might be without adequate technical support, for example, in night school.

We also all subscribed to a design that afforded maximum student control within the product with the goal of allowing as much practice as the student desired. As a result of Statan's careful planning, the student can reach any part of the program in 2-3 mouse clicks: select a unit/topic, click on an exercise, and if necessary, click on a help screen or return to the main menu. While the units are constructed in a roughly ascending order of exercise difficulty, a student may start at any point in the $C D$, and with any unit, and proceed either in the order implicit in the menu, or in any direction (see Figure 1). Within any unit, they could proceed step-by-step (using the blue arrows, see Figure 2), or skip around. On each screen, students are given maximum control of the learning process. For example, they can replay the video or photo sequence, repeating it as many times as desired (see Figure 2), or review each unit as often as they wish. All too often, computer software is designed so that once students have "mastered" a lesson or unit, they are not allowed to do it again. 
L.IVE ACTION EMGLISH InECRATIV
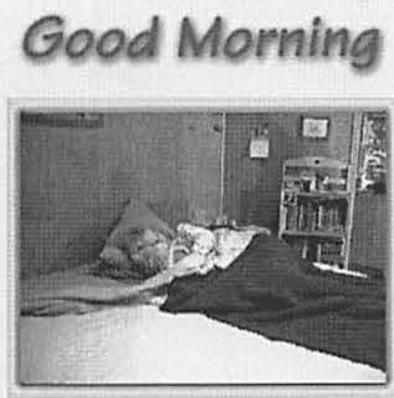

PLAY DI

QQ - MENU HELP QUIT

Figure 2. Watch from the Good Morning unit. The video is first seen and heard without text. Live Action English Interactive (Command Performance Language Institute 2000).

Learner control, frequent repetition, contextualized content, and multiple input channels were the main foundations of LAEI design. In the Watch activity (Figure 2), the student looks at the video or photo sequence and listens as the actions are performed; the video or photo sequences may be played as often as the student wishes. In Listen, the aural comprehension check following the Watch task, the student hears a sentence, clicks on the appropriate visual, hears the sentence repeated again, and sees the text only after the correct photo is clicked (see Figure 3). Thus, the "reward" for a correct response is reinforced input, as is the case throughout the program. We concluded from experience with other language learning CDs that some encouragement-such as Good, you've got it! or Sorry, try again - might be useful external motivation, but that the student should receive input repeatedly, whether answers were right or wrong. Additionally, feedback for incorrect answers should not be more attractive or compelling than feedback for correct answers. We consciously avoided the kind of negative feedback (i.e., the "raspberry" sound) that would make students want to click on an incorrect answer. Instead we put the rewards into having the student control the activities and make things happen. 


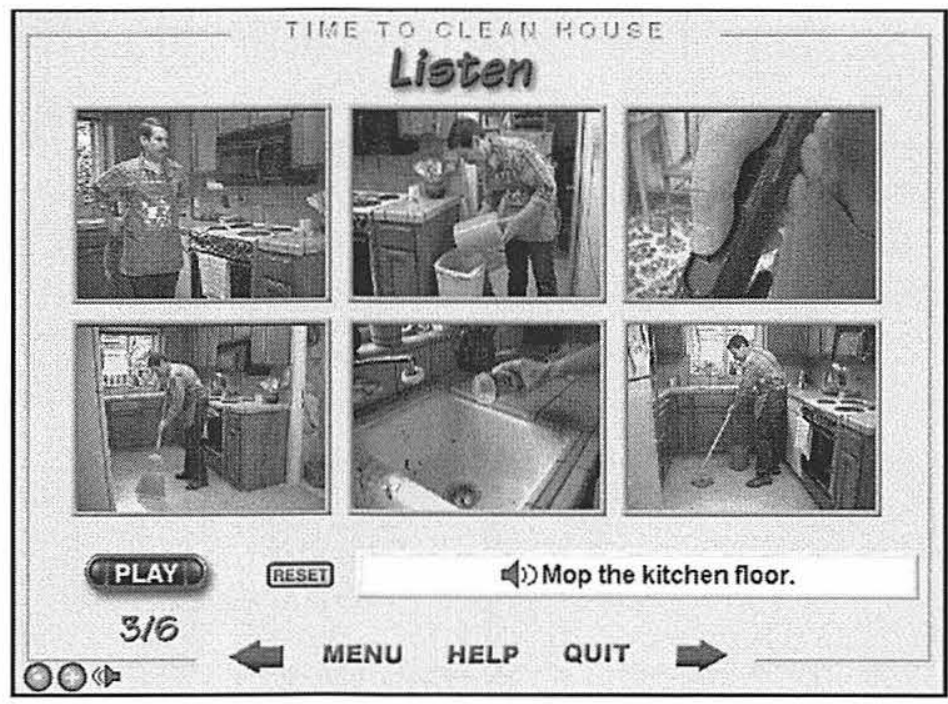

Figure 3. Listen activity for the Time to Clean House unit, Live Action English Interactive (Command Performance Language Institute 2000). The correct selection has been made, and the student can now read the sentence while hearing it again.

Students next encounter probably the most creative part of LAEI, the Interact task, the brainchild of Statan, our lead designer and program author. In this task, the student hears the command, performs an action with the mouse, and the command is then carried out on the screen with audio and video support. In some units, for instance, learners are asked to select the correct object and drag it to a photo on the screen. When they drop the object, it triggers the associated video segment or photo sequence, and the student hears the passage again. For example, in Good Morning, the student moves a newspaper to the screen, and the actress (actually one of our design team) reads it. In other units, the actor may speak to the user: Please give me the milk... Thank you! Where drag-and-drop is not used, the program brings to life the actions of different tools, e.g., a saw or a can opener, or even simulates driving a car (Figure 4). (Some students have referred to the program as the $C D$ that teaches you how to drive, which it definitely is not.) A large part of the motivational value here, and throughout the $C D$, is that the student makes the program run, and can do so as often as desired. The purpose of the $\mathrm{CD}$ is practice, not testing. 


\section{Best Practices}

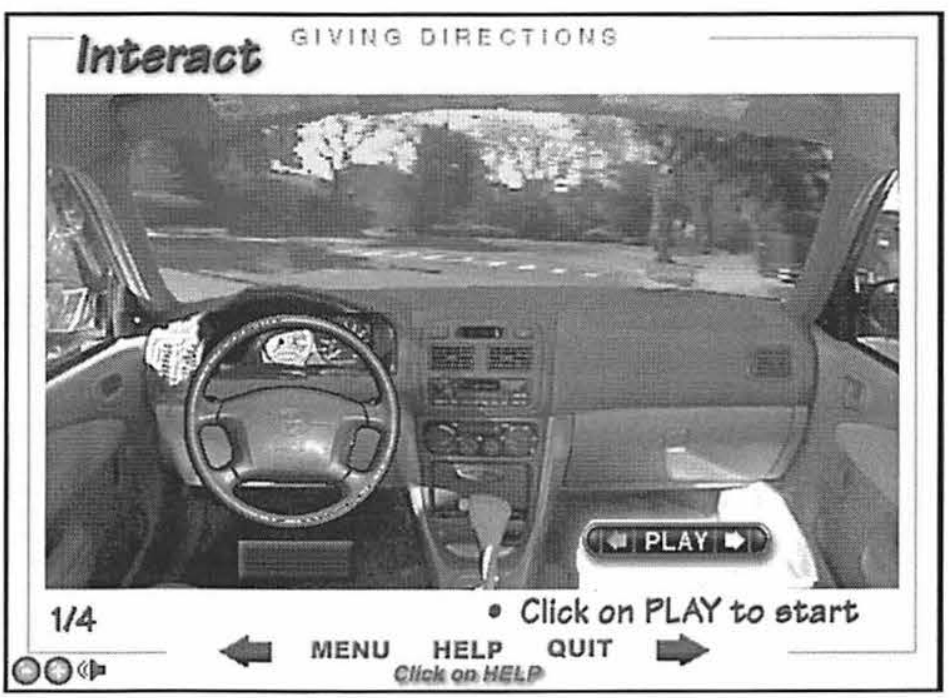

Figure 4. Interact screen from the Giving Directions unit: the student "drives" the car, following the verbal commands. Live Action English Interactive (Command Performance Language Institute 2000).

Following these opportunities for input and interaction, learners are introduced in Watch and Read to the entire passage in written form for the first time. They can either play the entire passage sequentially while reading and watching the video or photo sequence, or click on a particular sentence and watch the video for that segment alone. The student is then asked to interact with the sentences they have heard, read, and seen enacted by dragging and dropping parts of the script into their correct sequence in Order. The learner hears the sentence by clicking on it, and hears it again when it is dropped into place (see Figure 5 background).

Before continuing with a discussion of the last two parts of each unit, the more difficult activities, it should be mentioned that we decided to key all Help screens (with the exception of the Verbs, which will be discussed in a moment) to specific pages, and to make them primarily graphical/visual so that early learners, no matter what their native language, could understand them without resort to language that might be more complex than what was targeted in the lesson. We also had to assume that a teacher or tutor assisting the student might not be technically expert, or that a lab assistant might not be expert in English. Thus, Help screens in LAEI contain a minimum of verbal explanation (see Figure 5). In contrast, Help screens for LASI take into consideration that users would be fluent English speakers learning Spanish. 


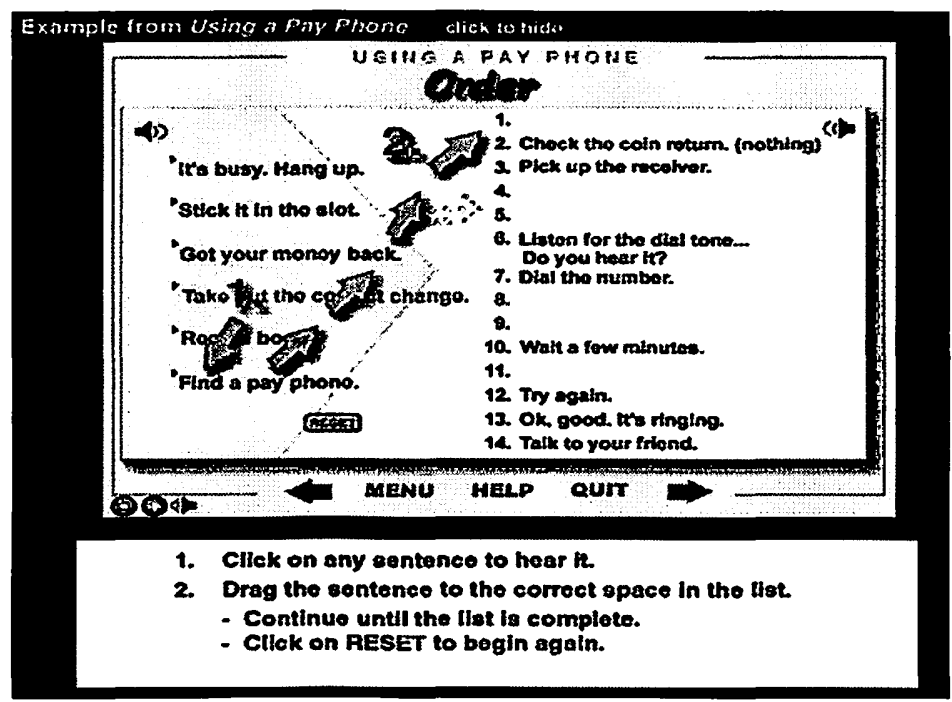

Figure 5. The graphical Help screen from Order, the drag-and-drop task in the Using a Pay Phone unit. Live Action English Interactive (Command Performance Language Institute 2000).

The Verb Help screens are an exception to our general rule of making help visually transparent. While the usual LAEI Help screen tells how the page operates, in Verb Help (accessed from the first screen of the Verbs task in each unit), we decided that we had to be fairly inclusive, since students might not be working with a grammar text at hand. At the same time, we intended the information to remain simple enough that a teacher's aide or lab assistant, who might not be trained in linguistics, could understand the explanations and perhaps help the student. We tried to avoid technical linguistics terminology or "grammar talk," insofar as possible. Thus, the Verb HeLP button leads to a full grammar section with tabs for particular aspects or tenses (see Figure 6). 


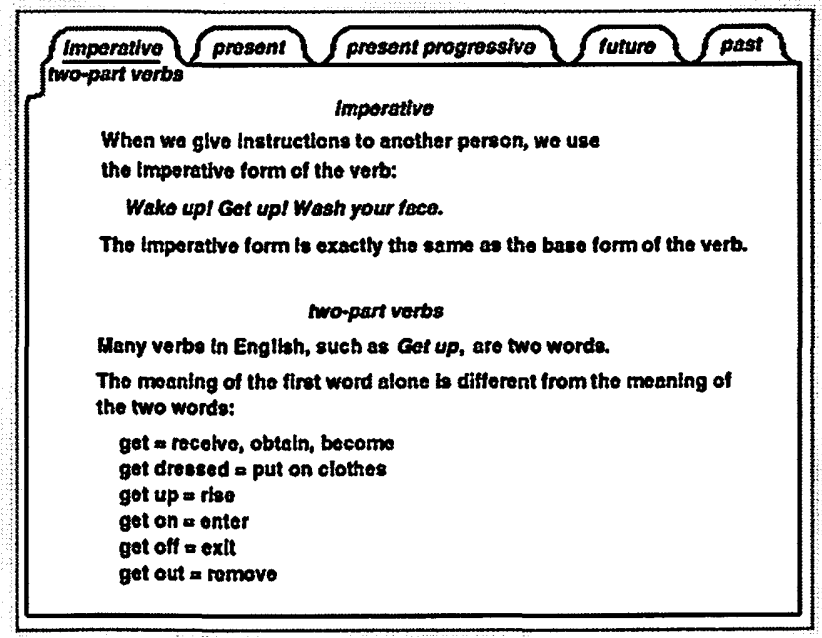

RETURN

Figure 6. The Verb Help screen with tabs to various grammatical tenses or aspects. Live Action English Interactive (Command Performance Language Institute 2000).

Practice for more advanced students represent a very wide range of language development (false beginner to advanced intermediate), we felt a need to include activities on the $\mathrm{CD}$ for more advanced students. The Verbs screens present the most challenging and time-intensive activities. Here the learners see the actions written in a variety of tenses as short narratives, rather than a sequence of commands. Each unit includes a screen with the simple past tense, because in English it is most often used for narration and has a number of commonly used irregular forms; and each also presents a screen with some form of the present, present progressive, or future progressive, and combinations of these as they would appear in spoken language. An effort was made to keep the language contextualized and as natural as possible. We wanted to avoid the mind-numbing problems of wholly de-contextualized sentences whose sole purpose was grammar practice.

In the Verbs activity, the student can listen to the whole text while reading it, and then click to perform a cloze exercise (Figure 7). Each time the student selects a blank to respond to, the computer reads the entire sentence aloud, thus ensuring plenty of listening practice even while the student concentrates on the grammar or spelling. While the present and future exercises are completed by drag-anddrop or clicking on the correct word or phrase, the past tense exercises require the learner to type in the verb correctly. Two-part verbs (e.g., put on, take off) are treated as one unit of meaning, and practice is also given with contractions, which are spoken naturally, but appear written out in their full form in the list of choices (see Figure 
7). Additional practice with the past tense screens can be obtained by asking students to hide the verbs to be typed, or having them to turn off the sound (using the SPEAKER icon on the screen), thus creating a pure reading-writing-grammar task. This one section of each unit encompasses hours of activity for the learner.

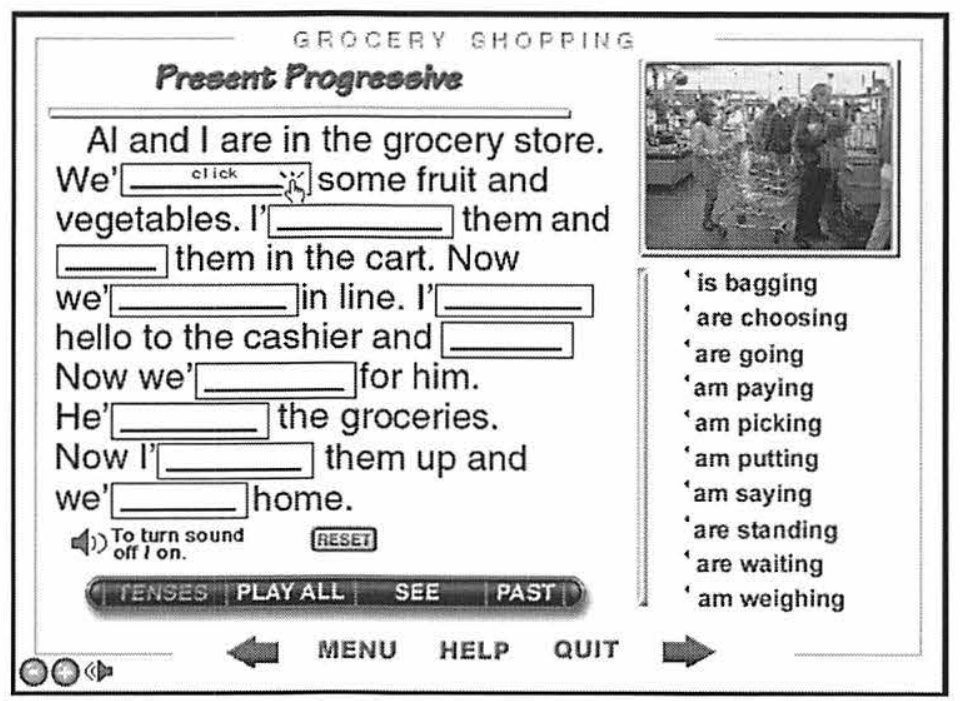

Figure 7. Reading and listening plus cloze exercise for Verbs in the Grocery Shopping unit. In the past tense task, the verbs may be hidden by clicking on their window and the sound may be turned off. Live Action English Interactive (Command Performance Language Institute 2000).

The final activity, Write, in each unit is a listening dictation. Here the software uses the spell-checker built into Director to help students correct errors. They can listen, type, check their answer, and receive hints from the program repeatedly (see Figure 8). Again, when the sentence is correctly typed, the software plays the video or photo segment as a reward, and the student hears the sentence again. 


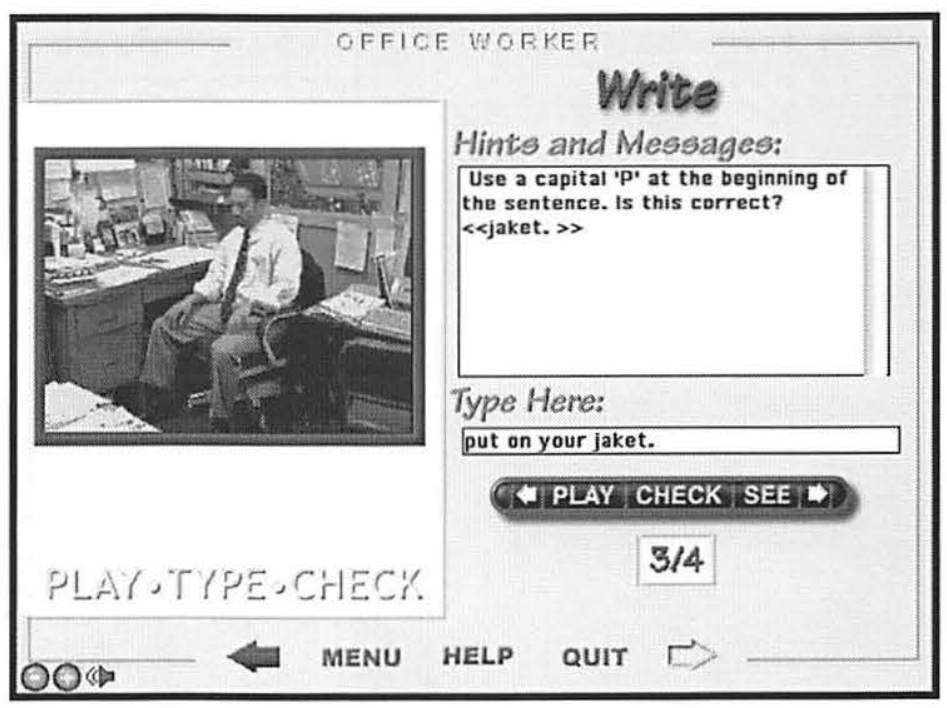

Figure 8. Write with an error hint from the Office Worker unit. A click on the SEE button allows a peek, but doesn't remain on the screen long enough to copy the answer. Live Action English Interactive (Command Performance Language Institute 2000).

As can readily be envisioned, the student has dozens of opportunities to receive input, without being bored by simple repetition. Even very low level students are encouraged by the format to work through the Verbs practice and listening dictation because the software is able to assist them and give them as many opportunities as needed to complete the task successfully. However, the teacher may want to tell true beginners to skip these two activities, Verbs and Write, until they feel ready to tackle them.

Since the $\mathrm{CD}$ is conceived of as a means to practice, rather than testing, the scoring system reflects in very simplistic fashion the learner's activity: students click on Score at the Main Menu (see Figure 1, bottom left corner) to see a record of how many tasks were attempted and how many were completed successfully (see Figure 9). We gave some thought here to the typical problems faced in adult education: many teachers will not have time to go to a networked computer, open a management system, type in a password, and look up student work; or students will be working at home or on public workstations, for instance, at a library, where records cannot be stored. Instead, at the Score screen, learners can simply fill in their name, click the PRINT button, and hand the page in to the teacher to be added to their portfolio or entered into a grade book. The teacher can quickly see which tasks were attempted and how many times they were practiced. Usually, the student will work on only one unit at a time, and when the program is closed, this record is not saved. On a second go at the unit, students will have a clean record. 
The Live Action Spanish product

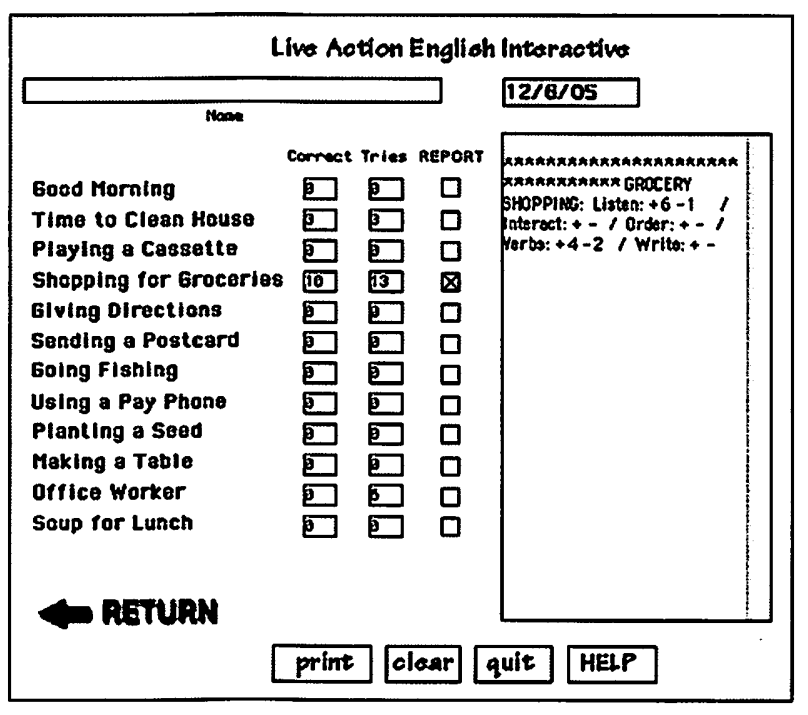

Figure 9. Score sheet with the Grocery Shopping unit reported. The student has completed 6 answers with one wrong in the Listen task. The student can also click the CLEAR button and start with a fresh slate without leaving the program. Scores are not saved and need not be accessed through a complicated password-secured system. Live Action English Interactive (Command Performance Language Institute 2000).

Translation of our $C D$ into another language besides English had been contemplated early on, since the Live Action text appears in 6 different languages. Our target population for LASI would be English speakers learning Spanish in the U.S., or possibly Spanish speakers mastering written forms of the language (a target audience in the U.S., where immigrant Spanish speakers are not always schooled in their own language), it was appropriate to retain the multi-ethnic characters evident throughout the LAEI CD. In addition to numerous Hispanic actors (portrayed for the most part by our students, not professionals), we also had many Asian faces and some blonde Euro-Americans as well. This ethnic mix reflects the reality of the U.S. context, particularly in our own students' lives.

A second consideration was technical: accents on the computer keyboard would demand the use of special keys or unusual keyboarding, and might differ depending on the type of computer. Statan had to solve the problem of how to include accents in Verbos and Escriba, where students would be asked to type input. He decided that this difficulty presented an opportunity to focus attention on accents in Spanish, which English speakers find particularly difficult. In Verbos, students are asked to decide if an accent is needed or not, but not where. In Escriba, the listening dictation, they have to select the cor- 
rect vowel to be accented (Figure 10). We felt that at the beginner level this solution raised awareness without overcorrecting the student—or requiring a special keyboard or unusual keystrokes.

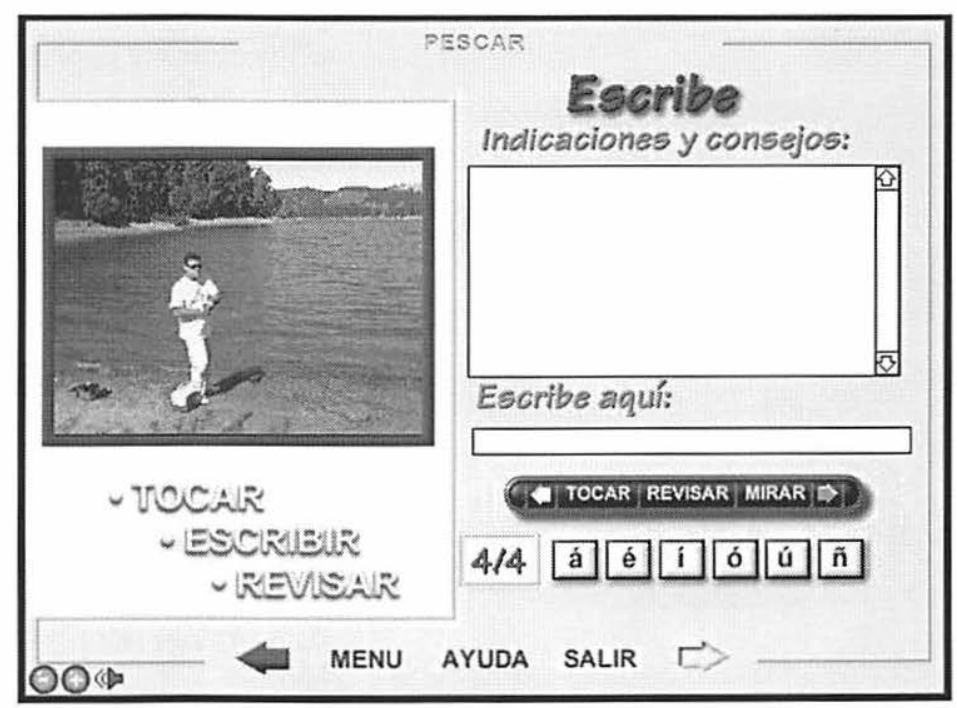

Figure 10. Escriba from the Pescar unit, with a choice of accent keys. Live Action Spanish Interactive (Command Performance Language Institute 2004).

Finally, as mentioned earlier, we decided in LASI to include a much more extensive grammar section and glossary in the Help screens, on the theory that English learners might be using the program as their sole source for language study.

\section{What we have learned}

First of all, we are very aware that while computers are ideal for additional language practice, the authoring programs and the capability of computers to handle natural language are both quite limited. In response to these limitations, we have tried to give students large amounts of practice under motivating conditions with contextualized language. Underlying some of the more imaginative aspects of our program, such as drag-and-drop video and driving a car simulation, is plenty of repetition, although we have tried not to all these to be the same old decontextualized drills that Roche (1999) laments. If getting the right answer is what computers do best, at least along the way we have made them respond to the students' wishes and needs.

Some important elements in this-or any-CD project are

-Humor - Many sections have a little built-in joke, such as the office worker who tries to fool his boss, and the cop's siren when the user runs the stop sign.

-Realism - The videos and photos are of people very 
Works Consulted much like the target students themselves (and many of them are, in fact, our students). They are seen in realistic settings (our own homes, the neighborhood grocery store), and they do not have all the polish of professional actors. This is the same appeal as so-called "reality television": people like to people-watch.

-Student control - Autonomy can be highly motivating for learners, and perhaps particularly so in language learning. A patient and dedicated student can listen repeatedly, leap to any part of the program with a click or two, perform the tests and activities multiple times without penalty, and focus most intently on whichever skills need the most work.

- Teacher interface - Teachers are an important element in using the software: They will know best which sections of the $C D$ to recommend to particular students and how best to integrate computer-based practice into their classroom activities. They can prepare students in class for the additional practice on the $C D$, and they can receive feedback on student work in print, a considerable time-saver for them.

-Understanding the limits of electronic media - Computers are best at affording additional practice, not teaching. Designers and teachers can, however, take good advantage of a well-designed computer program to give their students plenty of input.

The proof of this approach is in our students, who love the program. "Can we spend the night here?" pleaded one student in an evening class at City College of San Francisco the first time he used the program. He did not want to stop.

All Clear! Idioms in Context [Computer software]. 1994. Helen K. Fragiadakis and Larry Statan. Boston, MA: Heinle.

Asher, James. 1996. Learning another language through actions: The complete teacher's guidebook, 5th ed. Los Gatos, CA: Sky Oaks Productions.

Director MX 2004 [Computer software]. 2004. San Francisco, CA: Macromedia.

Glennan, Thomas. K., and Arthur Melmed. 1996. Fostering the use of educational technology: Elements of a national strategy. Santa Monica, CA: RAND. [MR-682-OSTP/ED] http://www.rand.org/publications/MR/MR682/contents.h tml (accessed May 30, 2005).

González, Dafne, and Arnold Mühren. 2004, March 10. Voice and Text Conference [Valencia, Spain, and Alkmaar, The 
Netherlands].

http://www.ilc.cuhk.edu.hk/english/tesol/2004/thurs.html (accessed July 8, 2004).

HyperCard, Version 2.4.1 [Computer software]. 1988. Cupertino, CA: Apple.

Krashen, Stephen D. 1982. Principles and practice in second language acquisition. Oxford: Pergamon Press.

Krashen, Stephen. D., and Tracy D. Terrell. 1983. The natural approach. Hayward, CA: Alemany Press.

Live Action English Interactive [Computer software]. 1999. Berkeley, CA: Command Performance Language Institute.

Live Action Spanish Interactive [Computer software]. 2004. Berkeley, CA: Command Performance Language Institute.

Making Connections, 1 [Computer software]. 1996. Larry Statan. Boston: Heinle

Oxford Picture Dictionary Interactive [Computer software]. 1996. New York: Oxford University Press. Available at http://www.esl.net/oxford_picture_dictionary_cdrom.html.

Ray, Blaine and Contee Seely. 1998. Fluency through TPR storytelling. 2nd ed. Berkeley, CA: Command Performance Language Institute.

Roche, Jörge. 1999. Multimedia in language instruction: Challenges and solutions. The IALL Journal of Language Learning Technologies, 31, No. 1/2: 45-52.

Romijn, Elizabeth K. and Contee Seely. 2002. Live Action English. Millennium ed. Berkeley, CA: Command Performance Language Institute.

Romijn, Elizabeth K. and Contee Seely. 1998. TPR is more than commands-At all levels. 2nd ed. Berkeley, CA: Command Performance Language Institute.

Seely, Contee, and Elizabeth K. Romijn. 2001. ;Viva la acción! Live Action Spanish, 2nd ed. Berkeley, CA: Command Performance Language Institute.

Takahashi, Noriko, and Maxine Frauman-Prickel. 1999. Action English pictures. Burlingame, CA: Alta Book Center.

Yeh, Aiden. Let's get physical! Students' presentation of lesson plans and activities: Video archive. 2004. http://www.geocities.com/tpr_videos/index.htm (accessed July 8,2004$)$. 
